\title{
Particularités de la reproduction des vaches allaitantes de races françaises
}

\author{
B. GRIMARD ${ }^{1}$, J. AGABRIEL ${ }^{2}$, G. CHAMBON 3 , A. CHANVALLON ${ }^{4}, F . C O N S T A N T^{1}$, S. CHASTANT \\ ${ }^{\prime}$ UMR BDR, ENVA, INRA, Université Paris Saclay, 78350, Jouy-en-Josas, France \\ 2 UMR1213 Herbivores INRA, Vetagro Sup, 63122, Saint-Genès-Champanelle, France \\ ${ }_{3}^{3}$ Clinique Vétérinaire Les Alouettes, pour la commission Vaches Allaitantes de la SNGTV, \\ 10 Quai Jules Chagot, 71300, Montceau les Mines, France \\ ${ }^{4}$ Institut de l'Élevage, UMR Biologie, Épidémiologie et Analyse de Risque en Santé Animale, \\ Oniris-INRA, CS40706, 44307, Nantes, France \\ ${ }_{5}^{5}$ École Nationale Vétérinaire de Toulouse, UMR Interactions Hôte-Pathogènes, INRA, ENVT, \\ 23 chemin des Capelles, 31876, Toulouse, France \\ Courriel : benedicte.grimard@vet-alfort.fr
}

Cet article valorise de nombreuses données françaises acquises sur la reproduction par les organismes de contrôle de performances ou dans les stations INRA. Les références sur les races françaises ont été privilégiées car ces races sont plus tardives que les races utilisées à l'étranger (Angus, Hereford, croisements de ces races avec des races laitières), ce qui amène parfois à présenter des données provenant de références anciennes et qui mériteraient d'être réévaluées dans les conditions d'élevage actuelles.

Les vaches allaitantes représentent plus de la moitié des femelles bovines présentes sur le territoire français (soit 4,05 millions, Institut de l'Élevage et Commission Nationale de l'Élevage 2015). Elles valorisent de nombreux territoires situés en zones défavorisées (Sanne et al 2013, Veysset et al 2014). Malgré les nombreux documents techniques annuels qui mettent en lumière les différences entre les caractéristiques de la reproduction de ces femelles par rapport aux races laitières, la reproduction des vaches allaitantes de races françaises reste mal connue, surtout pour les races rustiques, alors que leurs performances conditionnent grandement leur productivité (Offredo et Sibiluisky 2012). Aujourd'hui, si la taille moyenne des exploitations détenant des Vaches Allaitantes (VA) reste faible en France (26 VA en 2000, 33 en 2010, 32 en 2014), la part du cheptel détenu dans les exploitations de plus de 100 vaches augmente (11\% du cheptel en 2000, 20\% en 2010 , $28 \%$ en 2014 , Institut de l'Élevage et Commission Nationale de l'Élevage 2015). Ces grandes exploitations jouent un rôle majeur dans la restructuration du secteur. Or, pour maintenir le revenu et limiter les charges la maîtrise de la reproduction est cruciale : choix des périodes de vêlages, valorisation optimale des ressources fourragères, choix des périodes de vente, optimisation du travail à l'échelle de l'exploitation, diminution des périodes improductives. Cet article se propose de faire le point des connaissances actuelles concernant la reproduction des génisses et des vaches allaitantes en se focalisant sur les races françaises.

\section{1 / Productivité des vaches allaitantes}

La productivité des vaches allaitantes est mesurée par le nombre de veaux sevrés par vache présente. Le nombre de vaches présentes dans un élevage est calculé au prorata de leurs jours de présence sur la campagne (une vache présente dans l'élevage 6 mois sur 12 comptera pour 0,5 vache présente). Cette productivité était comprise entre 87,8 et $97,8 \%$ (97,8 veaux sevrés pour 100 vaches présentes) en 2013 pour les différentes races françaises (Bovins Croissance 2014). Elle est très variable au sein d'une race. Dans le quart supérieur des meilleurs élevages, elle était comprise la même année entre 105 et $110,3 \%$ soit 12,5 à 16,6 veaux sevrés de plus que la moyenne pour 100 vaches présentes (tableau 1). Les différences de productivité s'expliquent en grande partie par une moindre mortalité des veaux entre la naissance et le sevrage $(-2,5$ à - 4,9 points de pourcentage) mais aussi par une meilleure maîtrise de la reproduction. Dans le quart supérieur des meilleurs élevages le taux de renouvellement est légèrement plus élevé, ainsi que le \% d'Inséminations Animales (IA) réalisé mais surtout l'intervalle vêlage-vêlage moyen du troupeau est plus faible (- 4 à - 17 jours) aussi bien pour les multipares que pour les primipares (ce qui contribue à augmenter le nombre de vêlages par vache présente). L'amélioration de la productivité est aussi due à une réforme précoce des vaches vides ce qui permet de diminuer la durée des périodes improductives. La maîtrise de la reproduction est aussi associée à la diminution de la mortalité des veaux puisque celle-ci est plus faible dans les élevages qui pratiquent le vêlage groupé que pour ceux dans lesquels les vêlages sont étalés.

Il apparaît donc que des marges de progression sont possibles, notamment par une meilleure maîtrise de la reproduction, ce qui implique de tenir compte des particularités de ces génisses et vaches allaitantes.

\section{2 / Particularités de la repro- duction des génisses allaitan- tes}

\section{1 / Comportement}

Les génisses allaitantes sont peu manipulées entre la naissance et la mise à la reproduction. Pourtant les contacts dans le jeune âge ( 3 premiers mois) ou au moment du sevrage favorisent la docilité 
Tableau 1. Écarts de performances entre l'élevage moyen et le quart supérieur des meilleurs élevages classés sur la productivité globale (Élevages suivis par Bovins Croissance, Bovins Croissance 2014).

\begin{tabular}{|c|c|c|c|c|c|c|c|c|c|c|}
\hline \multirow{3}{*}{ Race } & \multirow{3}{*}{$\begin{array}{c}\text { Nombre } \\
\text { d'élevages }\end{array}$} & \multirow{3}{*}{$\begin{array}{c}\text { Productivité } \\
\text { globale } \\
\text { moyenne }\end{array}$} & \multirow{3}{*}{$\begin{array}{l}\text { Productivité } \\
\text { globale } \\
1 / 4 \text { supérieur }\end{array}$} & \multicolumn{7}{|c|}{ Ecarts de performances entre le quart supérieur et la moyenne des élevages } \\
\hline & & & & \multirow{2}{*}{$\begin{array}{c}\text { Vêlages } \\
\text { pour } 100 \\
\text { vaches } \\
\text { présentes }^{(b)}\end{array}$} & \multirow{2}{*}{$\begin{array}{l}\text { Premiers } \\
\text { vêlages } \\
(\%)\end{array}$} & \multirow{2}{*}{$\begin{array}{c}\text { Mortalité } \\
\text { avant } \\
\text { sevrage } \\
(\%)\end{array}$} & \multirow{2}{*}{$\begin{array}{l}\text { Veaux } \\
\text { nés } \\
\text { d'IA } \\
(\%)\end{array}$} & \multicolumn{3}{|c|}{$\begin{array}{l}\text { Intervalle vêlage-vêlage } \\
\text { (jours) }\end{array}$} \\
\hline & & & & & & & & $\begin{array}{l}\text { Trou- } \\
\text { peau }\end{array}$ & $\begin{array}{l}\text { Multi- } \\
\text { pares }\end{array}$ & $\begin{array}{l}\text { Primi- } \\
\text { pares }\end{array}$ \\
\hline Aubrac & 829 & 96,4 & 108,9 & 9 & 1,8 & $-2,5$ & 2,7 & -4 & -3 & -9 \\
\hline $\begin{array}{l}\text { Blonde } \\
\text { d'Aquitaine }\end{array}$ & 2112 & 87,8 & 105,9 & 14 & 4,4 & $-3,1$ & 0,1 & -16 & -15 & -18 \\
\hline Charolaise & 3623 & 94,9 & 108,7 & 9 & 1,6 & $-3,6$ & 5,8 & -6 & -5 & -9 \\
\hline Gasconne & 180 & 89,8 & 106,4 & 11 & 2,5 & $-3,8$ & 5,3 & -17 & -18 & -14 \\
\hline Limousine & 2710 & 94,1 & 107,7 & 11 & 3,7 & $-2,5$ & 1,6 & -8 & -7 & -12 \\
\hline Parthenaise & 256 & 88,8 & 105,0 & 10 & 1,7 & $-3,3$ & 3,3 & -6 & -7 & -4 \\
\hline $\begin{array}{l}\text { Rouge des } \\
\text { Prés }\end{array}$ & 230 & 91,8 & 108,1 & 10 & 1,9 & $-4,9$ & 2,6 & -12 & -11 & -12 \\
\hline
\end{tabular}

(a) Productivité globale $=$ Nombre de veaux sevrés pour 100 vaches présentes.

(b) Vache présente = Vache présente dans l'élevage au prorata du nombre de jours de présence sur la campagne.

Tableau 2. Âge et poids à la puberté dans trois races allaitantes.

\begin{tabular}{|c|c|c|c|c|c|}
\hline Race & Effectif & $\begin{array}{c}\text { Niveau } \\
\text { d'alimentation }\end{array}$ & $\begin{array}{c}\text { Age à la } \\
\text { puberté (mois) }\end{array}$ & $\begin{array}{l}\text { Poids à la } \\
\text { puberté (kg) }\end{array}$ & Références \\
\hline \multirow{5}{*}{ Charolaise } & 73 & 1 & 17,50 & 432 & Cobo et al (2013) \\
\hline & 310 & I & 14,20 & 418 & Mialon et al (1995) \\
\hline & \multirow{3}{*}{217} & Haut & $13,5^{a}$ & $401^{a}$ & \multirow{3}{*}{ Dozias et al (2006a) } \\
\hline & & Moyen & $15,2^{b}$ & $393^{a b}$ & \\
\hline & & Bas & $16,5^{\mathrm{c}}$ & $378^{\mathrm{b}}$ & \\
\hline \multirow{2}{*}{ Limousine } & 39 & Haut & $15,30^{\mathrm{a}}$ & 389 & \multirow{4}{*}{ D'Hour et al (1996) } \\
\hline & 38 & Bas & $17,10^{\mathrm{b}}$ & 384 & \\
\hline \multirow{2}{*}{ Salers } & 39 & Haut & $14,00^{\mathrm{a}}$ & 381 & \\
\hline & 38 & Bas & $15,00^{b}$ & 377 & \\
\hline
\end{tabular}

Au sein d'une même étude, les moyennes dont les exposants ne comportent aucune lettre en commun sont significativement différentes $(p<0,05)$.

des animaux par la suite (Boivin et al 1996, Boivin et al 2003). Ils sont à recommander si l'on souhaite pouvoir intervenir au premier vêlage en limitant les risques pour l'éleveur.

\section{2 / Âge à la puberté}

L'âge à la puberté est fortement influencé par le développement corporel comme pour les génisses laitières, mais les races allaitantes sont plus tardives. Les génisses laitières sont généralement pubères entre 9 et 12 mois selon les races lorsqu'elles ont atteint $40-45 \%$ du poids adulte (Le Cozler et al 2009). Dans les races allaitantes, la puberté apparaît plutôt entre 14 et 17 mois (tableau 2) lorsque les génisses ont atteint 50-55\% du poids adulte (Troccon et Petit 1989). L'âge à la puberté étant fortement lié au poids vif, l'apparition précoce de la puberté est conditionnée par les courbes de croissance sous la mère, puis pendant l'hiver qui suit le sevrage (voir niveaux d'alimentation haut/moyen/bas du tableau 2). L'âge à la puberté est aussi lié à la saison de naissance : les génisses nées à l'automne sont plus précoces que celles nées au printemps (Schillo et al 1992). L'élevage à proximité d'un mâle permet de diminuer l'âge à la puberté (Roberson et al 1991). Dans un élevage, choisir les femelles les plus précoces, c'est à dire les génisses pubères le plus tôt ou les plus lourdes à âge type permet de diminuer l'intervalle entre le vêlage et la reprise de cyclicité postpartum : en effet, ces paramètres sont génétiquement liés (Mialon et al 2000).

Selon Phocas et Sapa (2004), seules 30, 60 et $65 \%$ des génisses Limousines, Charolaises et Blondes d'Aquitaine respectivement sont cyclées à 15 mois (données sur plus de 3000 génisses par race suivies en station de contrôle des qualités maternelles sur descendance). De plus, la fertilité lors de la première mise à la reproduction est supérieure si l'on insémine 2 à 3 cycles après le $1{ }^{\text {er }}$ œstrus, plutôt que sur le $1^{\text {er }}$ cycle (Byerley et al 1987). Cette faible précocité sera donc une limite pour un premier vêlage à 2 ans, pratique néanmoins assez peu répandue en élevage allaitant en France sauf en race Blanc Bleu (tableau 3). Le premier vêlage à 2 ans impose donc une conduite alimentaire particulière des génisses (Institut de l'Élevage 2014) ou sera réservé aux jeunes femelles les plus lourdes et présentant le meilleur développement squelettique à 15 mois. Même si les animaux sont correctement sélectionnés sur ces 2 critères, la pratique du vêlage à 2 ans entraîne plus de difficultés de vêlage au premier vêlage, une mortalité des veaux légèrement plus élevée, une production laitière plus faible en première lactation et un poids à la réforme plus faible d'environ $30 \mathrm{~kg}$. Néanmoins, les performances sur l'ensemble de la carrière sont comparables à celles de génisses vêlant pour la première fois à 30 ou 36 mois (exemples en race Charolaise, Farrié et al 2008, Pierret et al 2009, Fortin et Coutard 2013). La pratique du vêlage à 2 ans peut entraîner une amélioration de la productivité liée 
Tableau 3. Performances de croissance et de reproduction des génisses de races à viande (données issues de 940150 vaches contrôlées dans 20849 troupeaux, 23 à $61 \%$ des effectifs français selon les races, Guerrier et Leudet 2014) et taux de premiers vêlages (10 600 troupeaux, Bovins Croissance 2014).

\begin{tabular}{|l|c|c|c|c|c|c|}
\hline Race & $\begin{array}{c}\text { Poids à } \\
\mathbf{1 2} \text { mois } \\
\mathbf{( k g )}\end{array}$ & $\begin{array}{c}\text { Poids à } \\
\mathbf{1 8} \text { mois } \\
\mathbf{( k g )}\end{array}$ & $\begin{array}{c}\text { Poids à 24 } \\
\text { mois (kg) }\end{array}$ & $\begin{array}{c}\text { Age au 1 } \\
\text { vêlage (mois) }\end{array}$ & $\begin{array}{c}\text { vêlages avant } \\
\text { 27 mois (\%) }\end{array}$ & $\begin{array}{c}\text { Taux de } \\
\text { premiers } \\
\text { vêlages (\%) }\end{array}$ \\
\hline Aubrac & $339 \pm 42$ & $429 \pm 42$ & $499 \pm 47$ & $35 \pm 3,8$ & 4 & 18,6 \\
\hline Bazadaise & $331 \pm 59$ & $414 \pm 71$ & $497 \pm 61$ & $38 \pm 5,1$ & 2 & $/$ \\
\hline Blanc Bleu & $335 \pm 52$ & $/$ & $/$ & $32 \pm 5,8$ & 27 & $/$ \\
\hline $\begin{array}{l}\text { Blonde } \\
\text { d'Aquitaine }\end{array}$ & $392 \pm 58$ & $500 \pm 66$ & $591 \pm 74$ & $36 \pm 4,6$ & 4 & 24,8 \\
\hline Charolaise & $387 \pm 53$ & $493 \pm 60$ & $588 \pm 64$ & $35 \pm 3,8$ & 5 & 26,1 \\
\hline Gasconne & $302 \pm 52$ & $400 \pm 57$ & $453 \pm 54$ & $36 \pm 4,4$ & 3 & 18,4 \\
\hline Limousine & $367 \pm 47$ & $465 \pm 58$ & $546 \pm 65$ & $35 \pm 3,9$ & 4 & 22,4 \\
\hline Parthenaise & $354 \pm 45$ & $446 \pm 52$ & $527 \pm 58$ & $35 \pm 4,2$ & 4 & 29,2 \\
\hline Rouge des Prés & $387 \pm 53$ & $486 \pm 61$ & $576 \pm 494$ & $34 \pm 4,8$ & 8 & 30,2 \\
\hline Salers & $347 \pm 41$ & $453 \pm 51$ & $527 \pm 47$ & $35 \pm 4,2$ & 6 & 20,6 \\
\hline
\end{tabular}

/ : Donnée manquante dans la race (faible nombre de troupeaux contrôlés sur le critère).

à une augmentation de 5 à $10 \%$ du nombre de vêlages dans le troupeau pour un effectif total comparable.

\section{3 / Objectifs de croissance}

Les performances de reproduction ultérieures sont liées au niveau alimentaire pendant toute la croissance de la génisse, de la naissance jusqu'au premier vêlage (Grenet et al 1996, Lassalas et al 1996, Dozias et al 2006a). Des niveaux d'alimentation faibles réduisent le poids au vêlage, la production laitière en première lactation et augmentent la durée de l'anœstrus postpartum après le premier vêlage. Des niveaux d'alimentation trop élevés entrainent de l'embonpoint, des difficultés de vêlage et en conséquence, de la mortalité des veaux.

Pour des génisses vêlant à 2 ans, les objectifs de croissance sont relativement élevés (Institut de l'Élevage 2014). Pour les races Charolaise, Limousine, Blonde d'Aquitaine et Rouge des Prés, un gain moyen quotidien (GMQ) supérieur à $1000 \mathrm{~g} / \mathrm{j}$ est nécessaire pendant la phase de croissance sous la mère. Les animaux doivent avoir atteint $60 \%$ du poids adulte à 1 an (GMQ 600 à $900 \mathrm{~g} / \mathrm{j}$ entre le sevrage et la mise à la reproduction) et $80 \%$ du poids adulte à la mise bas (GMQ 500 à $900 \mathrm{~g} / \mathrm{j}$ pendant la gestation en évitant les croissances fortes en fin de gestation).

Les objectifs à atteindre dans les différents types de conduite et l'intérêt économique du choix de l'âge au premier vêlage sont détaillés dans un rapport très bien étayé sur des données de terrain et disponible en ligne (Coutard 2011). Des courbes de croissance type sont données dans 4 grandes races allaitantes (Charolaise, Limousine, Blonde d'Aquitaine et Rouge des Prés) en fonc- tion de la date de naissance et de l'âge au vêlage des génisses.

Pour des génisses vêlant vers 30-33 mois, les objectifs sont élevés pendant la phase de croissance sous la mère (1 $000 \mathrm{~g} / \mathrm{j}$ au minimum) car les retards au sevrage sont difficilement rattrapés, mais les objectifs sont relativement faibles ensuite $(600$ à $700 \mathrm{~g} / \mathrm{j})$ d'autant que les animaux peuvent bénéficier du phénomène de croissance compensatrice à la mise à l'herbe (Blanc et al 2004).

Le vêlage à 36 mois est peu exigeant, il est accessible à tous les animaux avec des croissances modérées en hiver (400 à $600 \mathrm{~g} / \mathrm{j})$. Il est économe en concentrés. Il convient de plus de privilégier l'apport de fourrages grossiers dans l'alimentation des génisses afin de développer leur capacité d'ingestion. La complémentation ne fera appel qu'à de petites quantités de concentrés afin de réaliser des économies sur le poste alimentation du pré-troupeau. Par ailleurs, atteindre les objectifs de croissance recommandés pour les génisses suppose de bien mâ̂triser le parasitisme.

\section{4 / Mise à la reproduction}

Les génisses de race à viande doivent avoir atteint $70 \%$ du poids adulte à la mise à la reproduction (Institut de l'Élevage 2014), soit un pourcentage plus important que ce qui est recommandé pour les génisses laitières $(60 \%$ du poids adulte, Le Cozler et al 2009).

Lorsque le premier vêlage est tardif (entre 32 et 36 mois, tableau 3 ) le poids à la mise à la reproduction n'est généralement pas une limite. Les génisses ont atteint l'objectif préconisé vers 24 mois au moment de la mise à la reproduction.
Il se peut cependant que certaines génisses ne soient pas cyclées. C'est le cas si elles sont trop maigres (note d'état corporel $\leq 2$, Petit et Agabriel 1993) ou si l'on souhaite les mettre à la reproduction en hiver. Des données anciennes montrent en effet que certaines génisses, rentrées cyclées en stabulation, sont en anœstrus en hiver. Ceci serait lié au type de logement (exercice et éclairement, figure 1). Ces données mériteraient d'être réactualisées mais sont confirmées par les observations de terrain. Le recours à des traitements de maîtrise des cycles à base de progestérone ou de progestagènes permet d'induire les chaleurs et d'inséminer à l'aveugle sans détection des chaleurs (voir ci-après).

Le niveau de croissance autour de l'IA (0 vs $300 \mathrm{~g} / \mathrm{j}$ vs $700 \mathrm{~g} / \mathrm{j}$ ) n'a pas d'effet sur les performances de reproduction si les génisses sont suffisamment lourdes et en bon état corporel (Dozias et al 2006b).

La mise à la reproduction des génisses se raisonne plus en fonction de la (ou des) période(s) de vêlage que de l'âge au vêlage. Les génisses doivent être mise à la reproduction pour vêler en début de période pour des raisons de surveillance des vêlages mais aussi parce qu'elles auront un intervalle vêlage 1 -vêlage 2 (IVV) supérieur à celui des multipares (IVV supérieur de 5 à 15 jours à celui de la moyenne du troupeau dans les grandes races, (Guerrier et Leudet 2014). Dans le cas d'une double période de vêlage certaines génisses pourront être mises à la reproduction pour un premier vêlage à 30 mois ce qui améliore l'efficacité économique du système sans trop dégrader les performances zootechniques. S'il n'y a qu'une seule période de vêlage, les meilleures génisses seront mises à la reproduction pour un vêlage entre 32 
Figure 1. Relation entre type de logement et pourcentage de génisses cyclées : mesuré une fois en hiver (A), évolution au cours de l'hiver (B) (génisses Salers de 2 ans, d'après Aguer 1981).

\section{A}

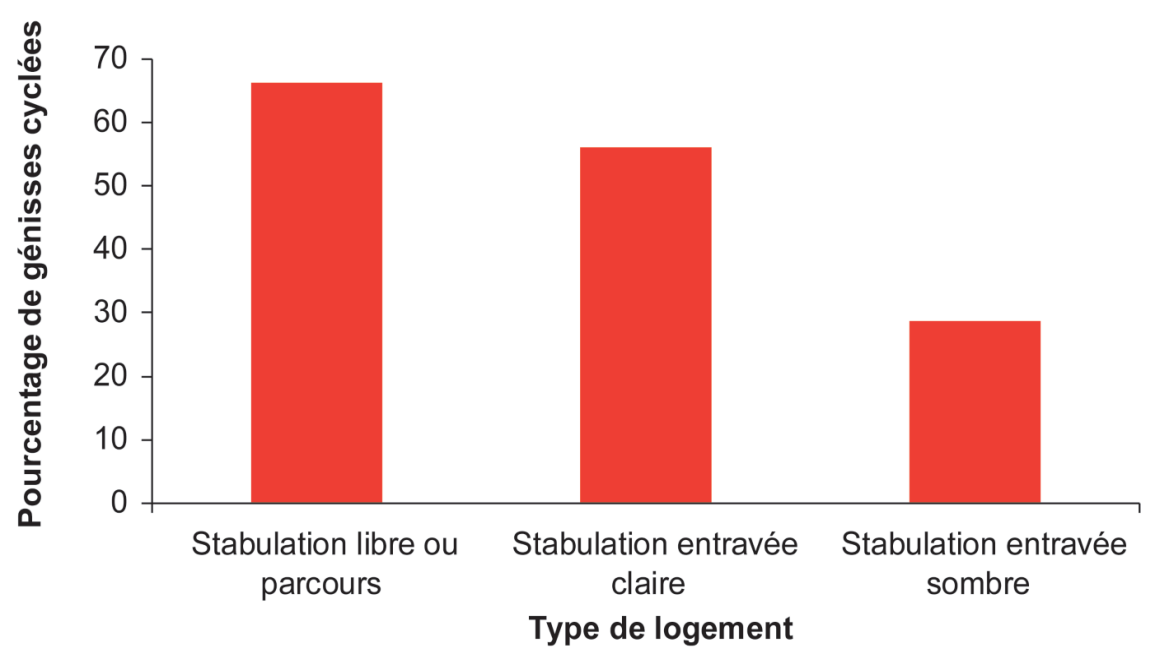

B

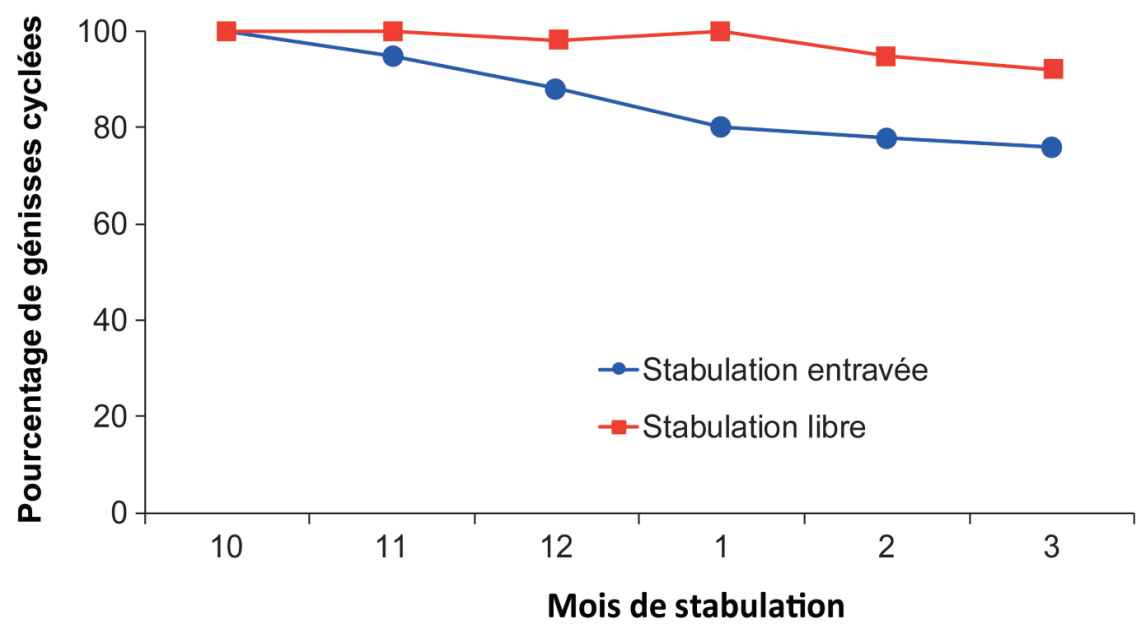

et 36 mois, 3 semaines avant la date moyenne de vêlage du troupeau. En cas de vêlages étalés on évitera les premiers vêlages trop tardifs (plus de 36 mois) encore fréquents mais coûteux dans les races allaitantes françaises (Coutard 2011).

Compte tenu de la bonne fertilité des génisses (taux de réussite en première IA supérieur à 50\%, Mounaix et al 2012) il est possible de ne les mettre à la reproduction que pendant une courte période ( 2 cycles) ce qui permet de sélectionner les génisses les plus fertiles.

\section{5 / Taux de renouvellement}

Le taux de premier vêlage varie de 18 à $30 \%$ dans les races allaitantes (tableau 3). Ces taux sont relativement faibles par rapport à ce qui est observé dans les races laitières. Ils sont plus élevés dans les élevages les plus productifs que dans d'une revue (Crowe et al 2014). La croissance folliculaire et la sécrétion de FSH («Follicle Stimulating Hormone ») reprennent précocement après vêlage chez la vache allaitante comme chez la vache laitière. Chez cette dernière, le premier follicule dominant ovule en l'absence de stress nutritionnel vers $10-15$ jours postpartum (figure 2). Par contre, chez la vache allaitante, la tétée et la présence du veau (contact visuel, olfactif, léchage) ont un effet central qui diminue la sécrétion de GnRH (« Gonadotropin Releasing Hormone ») et par conséquent de LH («Luteinizing Hormone ») : les vaches allaitantes en bon état corporel ne vont ovuler qu'après croissance et atrésie de 3 follicules dominants en moyenne, vers 30 jours postpartum (figure 2). Si l'état corporel est faible (moins de 2,5 au vêlage) plus de 10 vagues folliculaires vont se succéder avant la première ovulation qui n'interviendra que vers 70 à 100 jours postpartum (Stagg et al 1995).

La première ovulation, comme chez la vache laitière, est généralement silencieuse, c'est-à-dire non accompagnée d'œstrus, et suivie d'un cycle plus court que les suivants $(>70 \%$ des cas, Crowe et al 2014).

Après le vêlage, des anomalies de reprise de cyclicité sont observées. Chez la vache Prim'Holstein, les phases lutéales prolongées sont les plus fréquentes (sécrétion de progestérone élevée pendant plus de 19 jours, (Grimard et Disenhaus 2005)). Chez la vache allaitante, ce sont plutôt les retards de reprise de cyclicité qui sont observés (première ovulation après 50 jours postpartum, (Mann et al 2005, Disenhaus et al 2008)). Une fois la cyclicité établie, la fréquence des anomalies est faible.

Les facteurs de variation de la durée de l'anœstrus postpartum sont les mêmes chez la vache laitière et la vache allaitante (Humblot et Grimard 1996, Carthy et al 2014, Crowe et al 2014).

L'anœstrus postpartum est plus long d'environ 3 semaines chez les primipares que chez les multipares (Petit et Agabriel 1993, Ducrot et al 1994, Carthy et al 2014). Ceci est illustré au tableau 4 : l'IVV (Intervalle Vêlage-Vêlage) est plus long entre le premier et le deuxième vêlage que la moyenne observée pour tous les vêlages.

\section{3 / Reproduction des vaches allaitantes}

\section{1 / Ancstrus postpartum et reprise de cyclicité après vêlage}

L'anœstrus postpartum est plus long chez la vache allaitante que chez la vache laitière. Les mécanismes de reprise de la cyclicité après vêlage ont fait l'objet
La durée de l'anœstrus postpartum est très liée à la note d'état corporel au vêlage (Petit et Agabriel 1993, Yavas et Walton 2000b, Montiel et Ahuja 2005, Recoules et al 2013). Une note d'état corporel de 3 au vêlage et de 2,5 à la mise à la reproduction est recommandée pour les vaches qui vêlent à l'automne ou en début d'hiver et qui sont mises à la reproduction en 
Figure 2. Reprise de la croissance folliculaire et de la cyclicité chez la vache laitière (A : Exemple avec première ovulation 15 jours postpartum) et chez la vache allaitante (B : Exemple avec première ovulation 35 jours postpartum) correctement alimentées (d'après Crowe et al 2014).

La pulsatilité de la LH est représentée sur des fenêtres de $8 \mathrm{~h}$ (prise de sang toutes les $15 \mathrm{~min}$ ). Les cycles courts sont observés chez la majorité des vaches après première ovulation.

\section{A Vaches laitières}

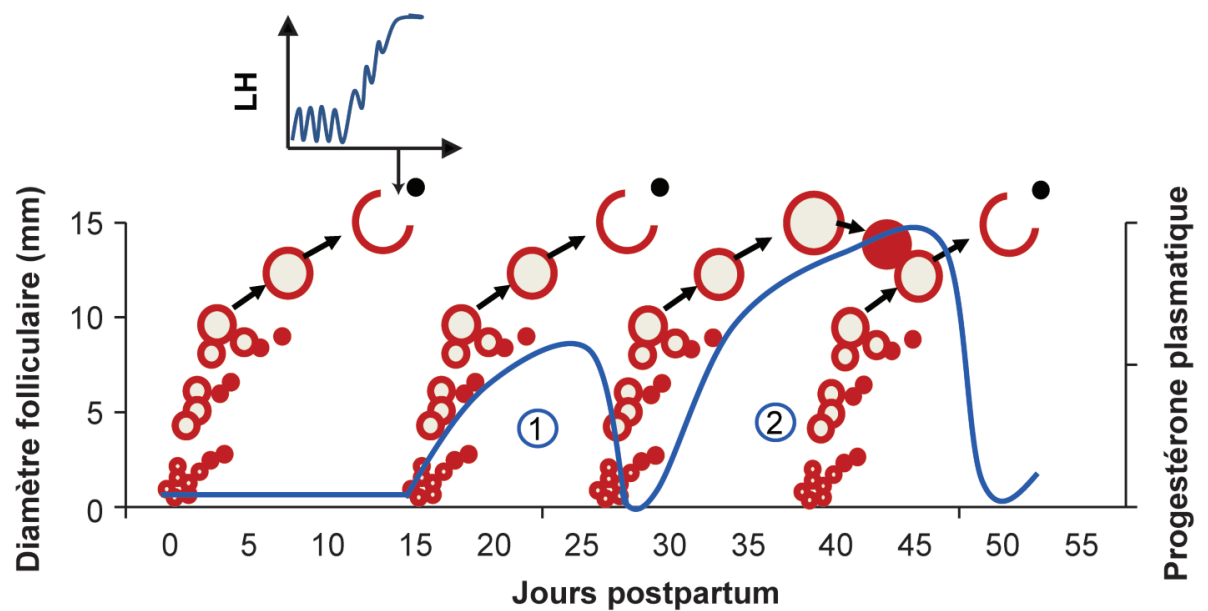

B Vaches allaitantes
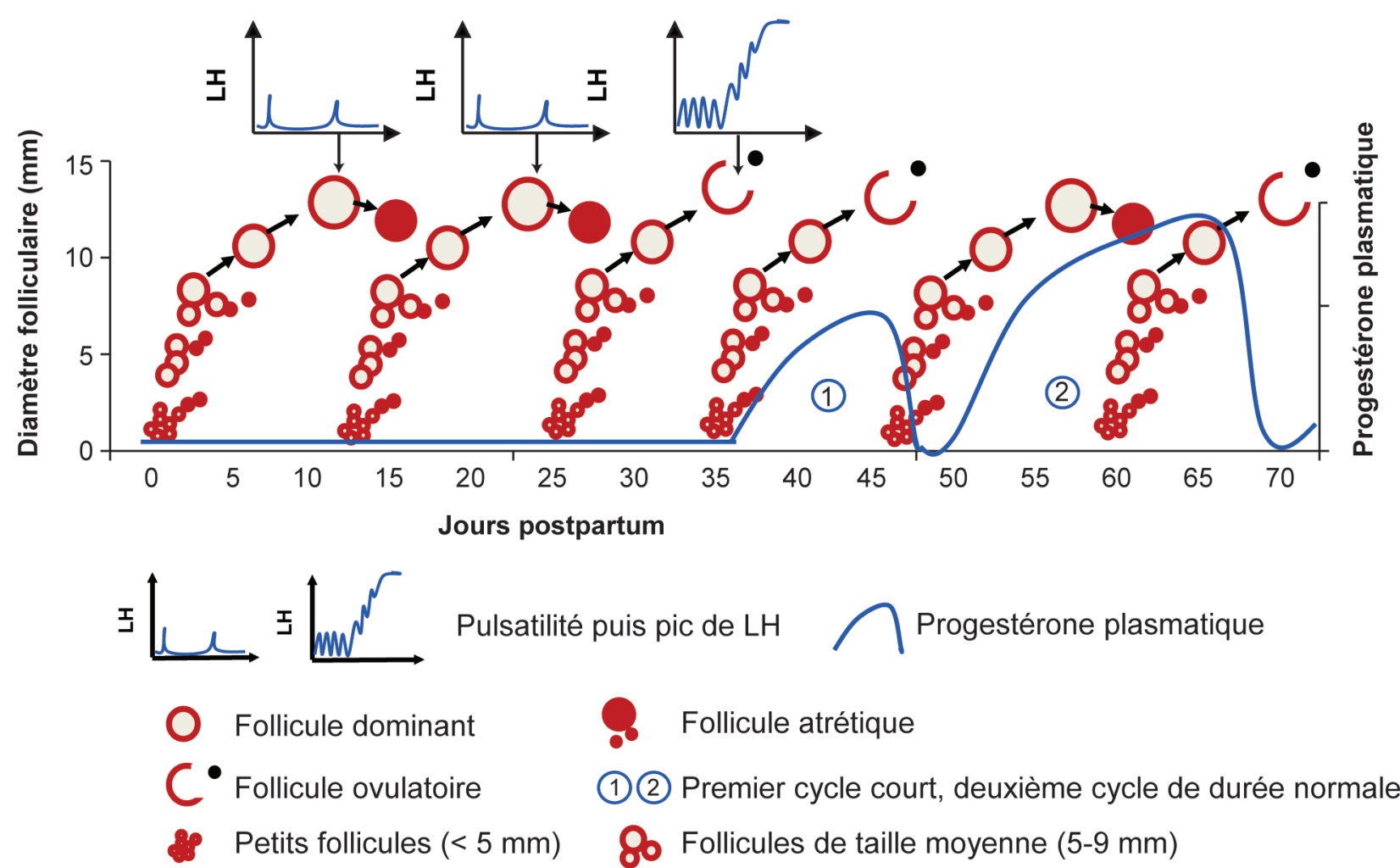

60-70 jours postpartum est significativement affecté dès qu'il y a intervention humaine, même si celle-ci est facile. Ces effets expliquent sans doute en partie les relations entre conditions de vêlage et intervalle vêlage-vêlage observées par Petit et Agabriel (1993) et Coutard et al (2007).

Le retour en chaleurs est plus précoce lors de vêlage d'automne que lors de vêlage d'hiver (70 à $80 \%$ de vaches cyclées 60-70 jours après un vêlage d'automne (Mialot et al 1998, Mialot et al 2003, Grimard et al 2007) contre 25 à $65 \%$ après un vêlage d'hiver (Ducrot et al 1994, Grimard et al 1994). Pour les vêlages d'hiver et de printemps, plus le vêlage est tardif, plus l'intervalle entre le vêlage et la reprise de cyclicité est court (Agabriel et al 1992, Blanc et Agabriel 2006).

Le retour en chaleurs est plus rapide pour les vaches qui n'allaitent pas leur veau (pratique utilisée dans certains cas
1994, Humblot et al 1996, Carthy 2014). Le pourcentage de vaches cyclées 
Tableau 4. Conditions de vêlage et intervalle vêlage-vêlage (IVV) moyen des vaches de races allaitantes (d'après Guerrier et Leudet 2014).

\begin{tabular}{|l|c|c|c|c|c|c|c|}
\hline \multirow{2}{*}{ Race } & \multirow{2}{*}{$\begin{array}{c}\text { Vaches } \\
\text { contrôlées }\end{array}$} & \multicolumn{4}{|c|}{ Conditions de vêlage (\%) } & \multicolumn{2}{c|}{$\begin{array}{c}\text { Intervalle vêlage-vêlage } \\
\text { (jours) }\end{array}$} \\
\cline { 3 - 8 } & & Facile & $\begin{array}{c}\text { Aide } \\
\text { facile }\end{array}$ & Difficile & Césarienne & IVV 1-2 & $\begin{array}{c}\text { IVV tous } \\
\text { Vêlages }\end{array}$ \\
\hline Aubrac & 60706 & 86 & 11 & 2 & 1 & $395 \pm 63,4$ & $382 \pm 56,1$ \\
\hline Bazadaise & 1879 & 82 & 13 & 4 & 1 & $441 \pm 102,5$ & $420 \pm 96,9$ \\
\hline Blanc Bleu & 3559 & 5 & 2 & 2 & 91 & $449 \pm 94,1$ & $444 \pm 91,1$ \\
\hline Blonde d'Aquitaine & 158351 & 77 & 17 & 4 & 2 & $417 \pm 79,3$ & $401 \pm 72,8$ \\
\hline Charolaise & 376098 & 67 & 25 & 5 & 4 & $395 \pm 58,6$ & $384 \pm 54,3$ \\
\hline Gasconne & 8068 & 95 & 3 & 1 & 0 & $432 \pm 96,3$ & $397 \pm 80,5$ \\
\hline Limousine & 248266 & 92 & 5 & 2 & 0 & $396 \pm 67,5$ & $384 \pm 60,2$ \\
\hline Parthenaise & 21359 & 66 & 25 & 6 & 3 & $395 \pm 66,8$ & $385 \pm 59,5$ \\
\hline Rouge des Prés & 18247 & 58 & 27 & 7 & 8 & $400 \pm 70,5$ & $388 \pm 63,0$ \\
\hline Salers & 50961 & 95 & 4 & 1 & 0 & $388 \pm 64,5$ & $379 \pm 57,8$ \\
\hline
\end{tabular}

lors de premier vêlage à 2 ans) que pour celles qui allaitent (Yavas et Walton 2000 b). La réduction de la fréquence des tétées (tétée 1 ou 2 fois par jour vs tétée ad libitum, (Stagg et al 1998)) est parfois citée comme un facteur pouvant réduire la durée de l'anœstrus mais cet effet semble nécessiter l'absence du veau entre les tétées (Crowe et al 2014). La réduction du nombre des tétées sera donc plus efficace en élevage de veaux sous la mère avec salle de tétée qu'en élevage allaitant en stabulation libre. Dans le premier cas les veaux sont logés à l'écart des mères, dans le deuxième les vaches continuent de voir, entendre et sentir leur veau dans les parcs qui leur sont réservés.

La mise en présence d'un mâle après le vêlage peut raccourcir l'intervalle vêlage-première ovulation (Yavas et Walton 2000a). L'effet est significatif si le mâle est introduit précocement après vêlage et est plus efficace chez les primipares que chez les multipares (Blanc et al 2002). L'effet mâle est nul si les vaches sont en bon état corporel au vêlage et semble dépendre de la saison de vêlage (Agabriel et al 2004).

\section{2 / Expression et détection des chaleurs}

Une partie des vaches allaitantes sont inséminées sur chaleurs observées. Il est alors nécessaire de bien connaître les signes d'œstrus afin de les détecter. Les données comportementales ont été récemment remises à jour dans les 3 grandes races allaitantes françaises Charolaise, Limousine et Blonde d'Aquitaine (118 œstrus observés, Blanc et al 2010).

Le signe le plus spécifique de l'œstrus est l'acceptation du chevauchement. Ce comportement est bien exprimé par les

Figure 3. Profil moyen comportemental moyen obtenu en phase œstrale chez des vaches allaitantes Blonde d'Aquitaine $(n=20)$, (Blanc et al 2010).

Comportements sexuels secondaires: Léchages et flairages sexuels, flehmen, appui du menton sur le dos ou la croupe d'une congénère.

Interactions sociales d'affinité : léchages et flairages non sexuels, jeux de tête. Interactions sociales agonistiques : menaces, coups de tête, lutte, évitement.

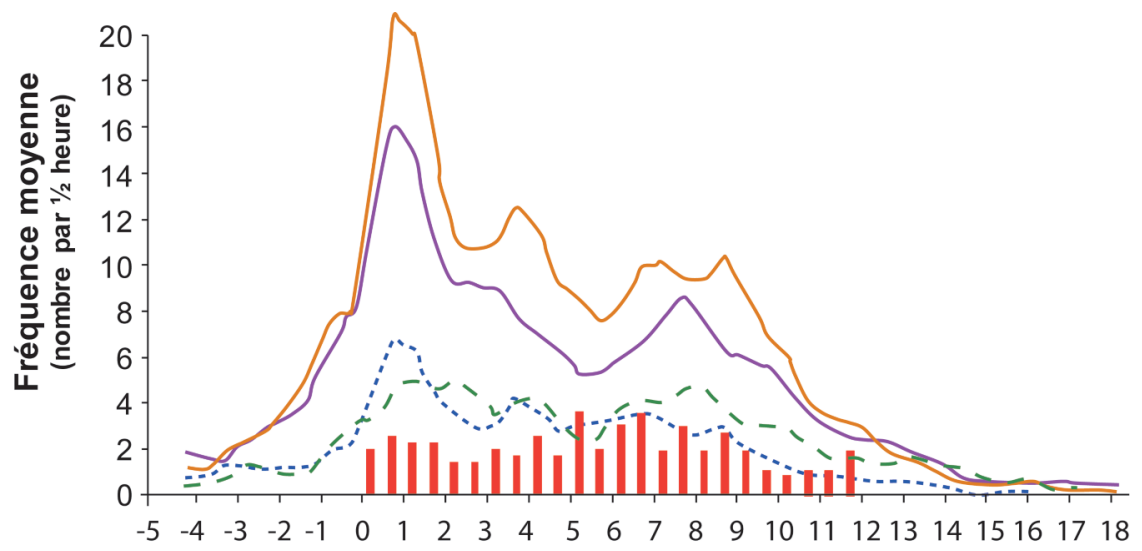

Heure par rapport au début de la 1ère acceptation de chevauchement observée

- Comportements sexuels secondaires

- - Chevauchements et tentatives de chevauchement

— Interactions sociales agonistiques

- - Interactions sociales d'affinité

Acceptations de chevauchement

vaches allaitantes (figure 3). On l'observe dans 90,93 et $100 \%$ des périodes de chaleurs en race Charolaise, Limousine et Blonde d'Aquitaine respectivement et les vaches expriment ce comportement entre 13 et 23 fois par œstrus. Il ne représente cependant que 3 à $7 \%$ des signes sexuels exprimés (autres signes sexuels : chevauchement, tentative de chevauchement, léchages et flairages sexuels réalisés vers la sphère génitale, Flehmen (retroussement de la lèvre supérieure), appuis du menton sur le dos ou la croupe d'un congénère). Si l'on tient compte de la période pendant laquelle les vaches acceptent le chevauchement, la durée des chaleurs des vaches allaitantes est comprise entre 6 et $10 \mathrm{~h}$. En tenant compte de tous les comportements sexuels la durée est de $11 \mathrm{~h}$ en race Limousine et Blonde d'Aquitaine et de $12 \mathrm{~h}$ en race Charolaise (Blanc et al 2010). Ces durées sont proches de celles observées sur les vaches allaitantes de race Angus, Hereford et croisements de ces races à l'étranger lors d'œstrus spontané (Hurnik et King 1987, Landaeta-Hernandez et al 2002, Landaeta-Hernandez et al 2006) mais sont plus courtes que celles observées sur des vaches taries ou après induction 
de l'œstrus (Landaeta-Hernandez et al 2002, White et al 2002, Floyd et al 2009).

Comme chez la vache laitière, la durée des chaleurs et le nombre d'acceptations du chevauchement augmentent avec le nombre de vaches en œstrus simultanément (Floyd et al 2009, Blanc et al 2010). Il sera donc plus facile de détecter les chaleurs lors de vêlage groupés que lors de vêlages étalés.

L'intensité de l'œstrus serait plus élevée entre 7 et $11 \mathrm{~h}$ du matin qu'aux autres périodes de la journée (Hurnik et King 1987, White et al 2002) mais cette distribution n'est pas retrouvée par tous les auteurs (Landaeta-Hernandez et al 2002).

La durée des chaleurs et le nombre d'acceptations du chevauchement seraient influencés par la saison. Pour White et al (2002), l'œstrus serait plus long en été qu'au printemps mais le nombre d'acceptations du chevauchement serait plus élevé en hiver qu'en été et au printemps. Cette dernière observation corrobore celle de Landaeta-Hernandez et al (2002) qui observent que le nombre d'acceptations du chevauchement diminue avec l'augmentation de la température ambiante.

La race, le rang de vêlage et le numéro d'œstrus ne semblent pas avoir d'effet sur la durée ni sur l'intensité de la manifestation des chaleurs (Hurnik et King 1987, Landaeta-Hernandez et al 2002, Blanc et al 2010).

La présence d'un taureau vasectomisé dans les lots de vache ne modifie pas le comportement d'œstrus (LandaetaHernandez et al 2006) mais peut grandement faciliter la détection de celui-ci si le taureau est équipé d'un harnais marqueur!

La sous-alimentation semble être favorable à l'expression des chaleurs chez les vaches cyclées : l'œstrus est plus long chez les vaches sous-alimentées ou en phase de mobilisation des réserves corporelles (+ 4 h environ, (Blanc et al 2010 , Recoules et al 2013)) et le nombre d'acceptations du chevauchement est doublé (Recoules et al 2013). Cet effet a aussi été observé chez des vaches laitières (Cutullic et al 2009).

À partir des enregistrements réalisés dans l'étude de Blanc et al (2010), les recommandations pour l'observation des chaleurs ont été actualisées (Beauchet 2010). La qualité de la détection (exactitude, sensibilité, spécificité) a pu être déterminée en fonction des signes observés (tableau 5). Ainsi, observer les animaux pendant 10 minutes au cours de 2 périodes calmes dans la journée permet de détecter $75 \%$ des vaches en chaleurs avec une sensibilité proche de $80 \%$, une spécificité de $97 \%$ et une exactitude de $88 \%$ si l'on prend en compte l'acceptation du chevauchement, le chevauchement ou au moins 3 signes sexuels secondaires (léchage ou flairage de la zone ano-génitale, Flehmen, appui du menton sur le dos ou sur la croupe d'une congénère).

\section{3 / Mode de reproduction}

Contrairement à ce qui est observé dans les races laitières, la monte naturelle domine dans les races allaitantes (tableau 6). Les veaux issus d'IA ne représentent que 3 à $24 \%$ des naissances selon les races (données estimées sur $88 \%$ des naissances issues de vaches allaitantes sur la campagne 2013). Le croisement, en IA ou en monte naturelle, est très répandu dans certaines races : $60 \%$ en race Salers, $41 \%$ en race Aubrac, $28 \%$ en race Gasconne. Une partie des veaux sont nés dans le même élevage que leur père (auto-renouvellement des taureaux, 8 à $31 \%$ des naissances).

Dans les troupeaux suivis par Bovins Croissance (25\% de l'effectif national), l'utilisation des taureaux de monte natu- relle est détaillée. Le taux de renouvellement moyen des taureaux est de $25 \%$ (18 à 35\% selon les races, tableau 7) ce qui montre que les taureaux sont réformés en moyenne tous les 4 ans afin de ne pas saillir leurs filles. Le pourcentage de taureaux ayant moins de 10 produits dans l'élevage est relativement élevé (37\% en moyenne) ce qui peut se justifier par la nécessité de faire de petits lots de vaches (génisses, petites parcelles, testage des taureaux sur de petits lots lors d'une première utilisation...), mais semble souligner un manque de productivité des taureaux puisque ceux-ci peuvent être mis avec 10 à 15 vaches dès la première année de mise à la reproduction vers 15 mois.

\section{4 / Fertilité}

La fertilité des vaches allaitantes en monte naturelle est peu documentée en France. Elle est supposée élevée. L'anœstrus postpartum serait le principal facteur limitant la fécondité.

Il n'existe pas, à notre connaissance, d'estimation standardisée de la fertilité des vaches allaitantes inséminées sur chaleurs observées. Lorsque cette fertilité est estimée par le taux de vêlage, comme pour les vaches laitières, et sur de grands nombres d'animaux (plusieurs dizaines de milliers par race), le taux de réussite en première IA des génisses est de 50 à $54 \%$ et celui des vaches de 48 à $52 \%$ dans les 3 grandes races françaises (Mounaix et al 2012).

Les traitements de maîtrise des cycles sont utilisables en élevage allaitant pour rationaliser le travail et grouper les vêlages. Ils permettent d'inséminer un groupe d'animaux à temps fixe, le même jour. Les protocoles à base de prostaglandines sont utilisables chez les génisses et les vaches cyclées, les protocoles associant GnRH et prostaglandines sont à réserver aux vaches de préférence cyclées.

Tableau 5. Bilan des scénarii à conseiller pour la détection des chaleurs des vaches allaitantes et qualité de détection associée (Chanvallon et al 2014).

\begin{tabular}{|c|c|c|c|c|c|c|c|}
\hline $\begin{array}{l}\text { Durée totale } \\
\text { d'observation } \\
\text { (min) }\end{array}$ & $\begin{array}{l}\text { Fréquence et durée } \\
\text { d'observation }(\mathrm{min})\end{array}$ & Moment d'observation & $\begin{array}{l}\text { Types de signes et nombre } \\
\text { d'observation par période }\end{array}$ & TD & SE & SP & E \\
\hline (inim) & $3 \times 5$ & $\begin{array}{l}\text { Entre } 2 \mathrm{~h} \text { et } 1 \mathrm{~h} \text { avant } \\
\text { l'alimentation du matin et du soir } \\
+ \text { dans l'heure qui suit } \\
\text { l'alimentation du matin }\end{array}$ & $\begin{array}{l}\text { Si } A C \geq 1 \text { ou chevauchements } \\
\geq 1 \text { ou signes secondaires } \geq 2\end{array}$ & 0,73 & 0,76 & 0,97 & 0,86 \\
\hline 20 & $2 \times 10$ & $\begin{array}{l}\text { Entre } 2 \mathrm{~h} \text { et } 1 \mathrm{~h} \text { avant } \\
\text { l'alimentation du matin et du soir }\end{array}$ & $\begin{array}{l}\text { Si } A C \geq 1 \text { ou chevauchements } \\
\geq 1 \text { ou signes secondaires } \geq 3\end{array}$ & 0,75 & 0,79 & 0,97 & 0,88 \\
\hline 30 & $2 \times 15$ & $\begin{array}{l}\text { Entre } 2 \mathrm{~h} \text { et } 1 \mathrm{~h} \text { avant } \\
\text { l'alimentation du matin et du soir }\end{array}$ & $\begin{array}{l}\text { Si } A C \geq 1 \text { ou chevauchements } \\
\geq 1 \text { ou signes secondaires } \geq 3\end{array}$ & 0,81 & 0,84 & 0,98 & 0,91 \\
\hline 45 & $3 \times 15$ & $\begin{array}{l}\text { Entre } 2 \mathrm{~h} \text { et } 1 \mathrm{~h} \text { avant } \\
\text { l'alimentation du matin et du soir } \\
+ \text { dans l'heure qui suit } \\
\text { l'alimentation du matin }\end{array}$ & $\begin{array}{l}\text { Si } A C \geq 1 \text { ou chevauchements } \\
\geq 1 \text { ou signes secondaires } \geq 3\end{array}$ & 0,85 & 0,88 & 0,96 & 0,92 \\
\hline
\end{tabular}

TD : Taux de Détection = Nombre de vaches détectées dans la journée/nombre de vaches en chaleurs $;$ SE $:$ SEnsibilité = Capacité à détecter les vaches effectivement en chaleurs. SP : SPécificité = Capacité à ne pas détecter les vaches qui ne sont pas en chaleurs ; $\mathrm{E}$ : Exactitude = Taux d'animaux correctement identifiés (en chaleurs ou non). 
Tableau 6. Utilisation de la monte naturelle, de l'Insémination Animale (IA) et du croisement dans les races allaitantes (données issues des naissances de paternité connue sur la campagne 2013, Miller 2014).

\begin{tabular}{|l|c|c|c|c|c|c|}
\hline Race & $\begin{array}{c}\text { Nombre de } \\
\text { naissances }\end{array}$ & $\begin{array}{c}\text { Nombre de } \\
\text { troupeaux }\end{array}$ & $\begin{array}{c}\text { \% Veaux de } \\
\text { paternité connue }\end{array}$ & $\begin{array}{c}\text { \% } \\
\text { Croisement }\end{array}$ & $\begin{array}{c}\text { \% Veaux } \\
\text { issus d'IA }\end{array}$ & $\begin{array}{c}\text { \% Auto- } \\
\text { renouvellement }\end{array}$ \\
\hline Aubrac & 162403 & 7232 & 91 & 41 & 9 & 11 \\
\hline Bazadaise & 2622 & 309 & 94 & 9 & 23 & 20 \\
\hline Blanc Bleu & 13731 & 1534 & 88 & 16 & 26 & 31 \\
\hline Blonde d'Aquitaine & 460127 & 23368 & 85 & 3 & 6 & 12 \\
\hline Charolaise & 1547137 & 44809 & 94 & 28 & 14 & 8 \\
\hline Gasconne & 16032 & 1101 & 78 & 22 & 3 & 16 \\
\hline Hereford & 1104 & 106 & 92 & 4 & 8 & 6 \\
\hline Limousine & 998417 & 34064 & 88 & 2 & 22 & 9 \\
\hline Parthenaise & 39925 & 1409 & 93 & 12 & 18 & 11 \\
\hline Rouge des Prés & 39147 & 2366 & 87 & 60 & 6 & 9 \\
\hline Salers & 200473 & 8693 & 86 & 86 & 13 & 19 \\
\hline $\begin{array}{l}\text { Support races } \\
\text { croisées }\end{array}$ & 341898 & 63086 & 79 & $\mathbf{1 6}$ & $\mathbf{1 2}$ & 17 \\
\hline Total & $\mathbf{3 4 5 9 1 7}$ & $\mathbf{1 2 1 8 7 5}$ & $\mathbf{8 8}$ & & $\mathbf{1 0}$ \\
\hline
\end{tabular}

${ }^{(*)}$ Veaux nés dans le même élevage que leur père.

Tableau 7. Utilisation des taureaux de monte naturelle dans les élevages suivis par Bovins Croissance (Guerrier et Leudet 2014).

\begin{tabular}{|l|c|c|c|c|}
\hline Race & $\begin{array}{c}\text { Nombre de } \\
\text { troupeaux }\end{array}$ & $\begin{array}{c}\text { Nombre de taureaux } \\
\text { de monte naturelle }\end{array}$ & $\begin{array}{c}\text { \% de taureaux ayant } \\
\text { moins de 10 produits } \\
\text { dans l'élevage }\end{array}$ & $\begin{array}{c}\text { Taux de } \\
\text { renouvellement } \\
\text { des taureaux (\%) }\end{array}$ \\
\hline Aubrac & 1292 & 2849 & 41 & 27 \\
\hline Bazadaise & 95 & 112 & 52 & 18 \\
\hline Blanc Bleu & 171 & 140 & 67 & 35 \\
\hline Blonde d'Aquitaine & 4749 & 6240 & 39 & 22 \\
\hline Charolaise & 7830 & 15426 & 38 & 27 \\
\hline Gasconne & 247 & 375 & 42 & 25 \\
\hline Limousine & 5526 & 10191 & 31 & 27 \\
\hline Parthenaise & 473 & 904 & 40 & 27 \\
\hline Rouge des Prés & 546 & 730 & 38 & 31 \\
\hline Salers & 1294 & 2003 & 39 & $\mathbf{2 5}$ \\
\hline Total & $\mathbf{2 0 8 5 9}$ & $\mathbf{3 7 8 4 3}$ & $\mathbf{3 7}$ & \\
\hline
\end{tabular}

Mais comme certaines femelles, génisses ou vaches du groupe, sont susceptibles d'être non cyclées, les traitements à base de progestérone ou de progestagènes sont recommandés : ils permettent d'induire les chaleurs chez les vaches en anœstrus (Grimard et al 2003, Picard-Hagen et al 2011). Trois présentations sont disponibles actuellement en France : implants auriculaires (méthode Crestar $\mathrm{SO}^{\circledR}$, MSD, Beaucouzé) ou dispositifs vaginaux (Prid Delta ${ }^{\circledR}$, CEVA, Libourne ou CIDR $^{\circledR}$, Zoétis, Paris). Une injection de Buséréline (analogue de GnRH) est recommandée en début de traitement pour la méthode Crestar $\mathrm{SO}^{\circledR}$, une injection de prostaglandine $\mathrm{F}_{2 \alpha}$ ou analogue est réalisée en fin de traitement ainsi qu'une injection d'eCG (« equine Chorionique Gonado-tropin », 400 à 600 UI selon les races et le rang de vêlage). Un nouveau protocole a été testé récemment, il associait GnRH, progestagène et prostaglandines, l'injection finale d'eCG étant remplacée par une injection de GnRH. Les modes d'action de ces différents traitements sont détaillés dans les deux articles cités ci-dessus.

Ces traitements donnent de bons résultats lorsqu'ils sont utilisés chez animaux en bon état corporel, plus de 50 jours après vêlage et sur des vaches qui n'ont pas subi de césarienne : la fertilité, mesurée entre 35 et 60 jours après IA, est alors comprise entre 54 et $75 \%$ (tableau 8).

Les facteurs de variation de la fertilité à l'œstrus induit ont été détaillés par Grimard et al (2003) en utilisant de nom- breuses références françaises et ont fait l'objet d'une revue récente (Bo et Baruselli 2014).

La fertilité à l'œstrus induit augmente avec l'intervalle vêlage-traitement. Ainsi on conseille de respecter un intervalle vêlage-traitement d'au moins 50 jours lors d'induction/synchronisation des chaleurs. La fertilité à l'œstrus induit est supérieure de 5 à 10 points de pourcentage chez les vaches cyclées avant traitement par rapport aux vaches non cyclées. Elle est significativement affectée par le rang de vêlage, moins bonne chez les primipares que chez les génisses nullipares et les vaches multipares. La note d'état corporel au vêlage ou à la mise en place du traitement sont associées à la fertilité à l'œstrus induit : le taux de gestation 
Tableau 8. Fertilité à l'œstrus induite après utilisation de traitements de maîtrise des cycles à base de progestérone ou de progestagène chez la génisse et la vache allaitante.

\begin{tabular}{|c|c|c|c|c|}
\hline Type d'animaux & Nombre & Traitement & $\begin{array}{c}\text { Taux de gestation* } \\
(\%)\end{array}$ & Référence \\
\hline \multirow{2}{*}{ Génisses Charolaises } & 126 & $\begin{array}{l}\text { Implant Crestar } \mathrm{SO} \otimes 10 \text { jours } \\
\text { GnRH à la pose } \\
\text { PGF2a } 2 \text { jours avant retrait } \\
\text { eCG au retrait } \\
\text { IA } 48 \mathrm{~h} \text { après retrait }\end{array}$ & 61,9 & \multirow{3}{*}{ Grimard et al (2007) } \\
\hline & 126 & $\begin{array}{l}\text { Implant Crestar } \mathrm{SO} \otimes 10 \text { jours } \\
\text { GnRH à la pose } \\
\text { PGF2 } 2 \text { jours avant retrait } \\
\text { eCG au retrait } \\
\text { IA } 36 \text { h après retrait }\end{array}$ & 58,7 & \\
\hline $\begin{array}{l}\text { Vaches Limousines et } \\
\text { Blondes d'Aquitaine }\end{array}$ & 69 & $\begin{array}{l}\text { Implant Crestar } \mathrm{SO} \otimes 10 \text { jours } \\
\text { GnRH à la pose } \\
\text { PGF2 } 2 \text { jours avant retrait } \\
\text { eCG au retrait } \\
\text { IA } 48 \mathrm{~h} \text { après retrait }\end{array}$ & 75,4 & \\
\hline Vaches allaitantes & 465 & $\begin{array}{l}\text { Prid@ } 7 \text { à } 9 \text { jours } \\
\text { PGF2 } 24 \mathrm{~h} \text { avant retrait } \\
\text { eCG } 400 \text { à } 600 \mathrm{Ul} \text { au retrait }\end{array}$ & 65,5 & Floch et al (2008) \\
\hline Génisses allaitantes & 132 & $\begin{array}{l}\text { Prid Delta }{ }^{\circledR} 7 \text { ou } 9 \text { jours } \\
\text { PGF2a } 24 \text { h avant retrait } \\
\text { eCG } 400 \text { Ul au retrait } \\
\text { IA } 48 \text { ou } 56 \text { h après retrait }\end{array}$ & 59,8 & \multirow{2}{*}{ Gatien (2010) } \\
\hline Vaches allaitantes & 101 & $\begin{array}{l}\text { Prid Delta } ₫ 9 \text { jours } \\
\text { PGF2 } 24 \mathrm{~h} \text { avant retrait } \\
\text { eCG } 400 \text { à } 600 \text { Ul au retrait } \\
\text { IA } 48 \text { ou } 56 \mathrm{~h} \text { après retrait }\end{array}$ & 65,3 & \\
\hline Génisses Limousines & 55 & \multirow{3}{*}{$\begin{array}{l}\text { CIDRß } 7 \text { jours } \\
\text { PGF2a } 24 \text { h avant retrait } \\
\text { eCG } 400 \text { à } 600 \text { Ul au retrait } \\
\text { IA } 56 \text { h après retrait ou sur } \\
\text { chaleurs observées }\end{array}$} & 58,2 & \multirow{3}{*}{ Meli (2009) } \\
\hline Vaches Limousines & 95 & & 53,9 & \\
\hline $\begin{array}{l}\text { Vaches et génisses } \\
\text { Charolaises }\end{array}$ & 72 & & 54,2 & \\
\hline \multirow{2}{*}{$\begin{array}{l}\text { Vaches Aubrac, } \\
\text { Blondes d'Aquitaine, } \\
\text { Charolaises et } \\
\text { Limousines }\end{array}$} & 78 & $\begin{array}{l}\text { GnRH à la pose, implant Crestar } \\
\text { SO } 7 \text { jours, PGF2 } \alpha \text { au retrait, } \\
\text { GnRH à J9, IA J10 }\end{array}$ & 53,8 & \multirow{2}{*}{ Esqurial et al (2014) } \\
\hline & 78 & $\begin{array}{l}\text { GnRH à la pose, implant Crestar } \\
\text { SO } 9 \text { jours, PGF2 } \alpha \text { à J7, eCG } \\
\text { à J9, IA J11 }\end{array}$ & 60,3 & \\
\hline
\end{tabular}

* Mesuré précocement par dosage de la PSPB, par échographie ou par palpation transrectale selon les études

est supérieur de 10 à 30 points chez les vaches en bon état corporel (note $\geq 2,5$ sur 5) par rapport aux vaches en mauvais état (note $<2,5)$. La pratique du «flushing » (2 UF/jour supplémentaires pendant le traitement et pendant 3 semaines après IA) améliore de 5 à 30 points la fertilité des vaches maigres. Un sevrage temporaire du veau permet d'améliorer la fertilité des vaches maigres mais celuici doit durer au moins 48 h et est généralement conseillé entre la fin du traitement et l'IA (Montiel et Ahuja 2005). Dans certaines études, la fertilité des animaux les plus légers et les plus lourds est inférieure à celle des animaux de la classe de poids intermédiaire. L'intervention au vêlage est associée à une diminution de la fertilité à l'œstrus induit. Après extraction forcée ou césarienne le taux de gestation est diminué de 15 à 30 points par rapport aux femelles ayant vêlé sans aide. Pour certains auteurs, même l'aide facile affecte significativement le taux de gestation après traitement. Dans les systèmes avec vêlage de fin d'automne-début d'hiver, la fertilité à l'œstrus induit est élevée en début de saison, elle baisse en fin d'hiver puis remonte si le traitement est mis en place après la mise à l'herbe. Par contre, si les vaches sont inséminées autour de la mise à l'herbe, le taux de gestation diminue et cela est associé à un taux d'urée dans le sang élevé, probablement lié au taux d'azote soluble de l'herbe jeune. Dans les troupeaux avec vêlage de fin d'été-début d'automne, le taux de cyclicité avant traitement est généralement élevé et la fertilité l'est aussi. Les facteurs de variation de l'efficacité des traitements de maîtrise des cycles sont donc nombreux avec, de plus, des effets cumulatifs. Deux choix sont alors possibles : ne réserver ces traitements qu'aux animaux ne présentant pas ou peu de facteurs de risque pour maximiser les résultats, ou utiliser ces traitements chez les animaux à risque mais en étant conscient qu'il s'agit plutôt d'une utilisation thérapeutique des traitements de synchronisation et que les résultats seront plus faibles que dans le premier cas.

\section{5 / Gestation et mise bas}

La durée de gestation moyenne est plus élevée dans les races allaitantes (281,6 à 295,6 jours, tableau 9) qu'en race Prim' Holstein (280,9 jours). Les données viennent d'être récemment actualisées (Ledos et Moureaux 2013). L'écart-type de la 
Tableau 9. Durée moyenne de gestation et fréquence de gestations multiples chez les principales races laitières et chez les races allaitantes d'après Ledos et Moureaux (2013).

\begin{tabular}{|c|c|c|c|c|}
\hline Race & Effectif & $\begin{array}{l}\text { Durée de gestation } \\
\text { moyenne (jours) }\end{array}$ & $\begin{array}{l}\text { Ecart-type } \\
\text { (jours) }\end{array}$ & $\begin{array}{c}\% \text { Gestations } \\
\text { multiples }\end{array}$ \\
\hline \multicolumn{5}{|l|}{ Laitières } \\
\hline Normande & 576534 & 286,6 & 6,4 & 3,5 \\
\hline Montbéliarde & 910714 & 287,6 & 6,2 & 4,1 \\
\hline Prim'Holstein & 3850213 & 280,9 & 6,1 & 3,4 \\
\hline \multicolumn{5}{|l|}{ Allaitantes } \\
\hline Aubrac & 13176 & 284,1 & 6,2 & 2,2 \\
\hline Bazadaise & 1087 & 290,3 & 6,2 & 2,1 \\
\hline Blanc Bleu & 5111 & 281,6 & 6,3 & 0,9 \\
\hline Blonde d'Aquitaine & 123107 & 295,6 & 6,3 & 2,1 \\
\hline Charolaise & 337422 & 287,5 & 6,4 & 4,8 \\
\hline Gasconne & 2500 & 288,5 & 5,8 & 4,0 \\
\hline Limousine & 109136 & 290,7 & 6,3 & 1,6 \\
\hline Parthenaise & 18702 & 287,8 & 6,2 & 2,2 \\
\hline Rouge des Prés & 14602 & 287,8 & 6,5 & 4,3 \\
\hline Salers & 12335 & 286,6 & 5,9 & 2,4 \\
\hline
\end{tabular}

durée de gestation moyenne est relativement élevé, 5 à 6 jours, ce qui signifie que $95 \%$ des animaux fécondés le même jour vêleront sur une plage de 20 à 24 jours. Dans toutes les races, la gestation est plus courte lors de gestations multiples que lors de gestations simples ( -4 à -6 jours) et un peu plus courte (- 1 jour) pour la première gestation que pour les suivantes (valeur significative sur de grands nombres d'animaux mais peu utile en pratique). Le taux de gémellité atteint $4 \%$ dans 3 races : Charolaise, Gasconne et Rouge des Prés. La prolificité, même si elle permet d'améliorer un peu la productivité en élevage allaitant, n'est pas recherchée en raison des difficultés de vêlages liées à la présence de jumeaux et au free-martinisme (les femelles jumelles de mâles sont la plupart du temps subfertiles ou infertiles).

Certaines races allaitantes présentent des taux élevés de vêlages difficiles. C'est le cas en races Blanc Bleu, Rouge des Prés, Charolaise et Parthenaise pour lesquelles les taux de vêlages difficiles et par césarienne sont respectivement de 93, 15, 9 et $9 \%$ (tableau 5). Si ces taux élevés sont acceptés en race Blanc Bleu, la sélection des taureaux à bonne valeur génétique pour la facilité de naissance est un objectif dans les 3 autres races mentionnées. En effet, le vêlage difficile a un effet délétère sur les performances de reproduction ultérieures (augmentation de l'intervalle vêlage-vêlage), la mortalité des veaux et la longévité des vaches (Coutard et al 2007, Coutard 2011).

\section{6 / Endométrites}

Les endométrites, fréquentes chez la vache laitière, ont été très peu étudiées chez la vache allaitante.

L'inflammation utérine est très intense dans les quinze premiers jours postpartum. Si elle persiste au-delà de 21 jours après le vêlage à un niveau trop élevé, elle est délétère pour les performances de reproduction (LeBlanc 2014). Cette inflammation utérine exagérée au-delà de 21 jours post-partum, dénommée endométrite, prend deux formes : la forme clinique qui se manifeste par des écoulements vaginaux purulents, et la forme subclinique, pour laquelle aucun écoulement n'est présent et ne se traduisant que par la présence d'une proportion anormalement élevée de leucocytes sur un frottis endométrial (cytobrosse ou lavage utérin). Les formes subcliniques sont les plus fréquentes.

Comme chez la vache laitière, la proportion de vaches atteintes d'endométrite serait élevée dans les 50 premiers jours postpartum chez les vaches allaitantes (82\%, Santos et al 2009; 77\%, Ricci et al 2015) et diminuerait ensuite (17\%, Santos et al 2009; 23\%, Ricci et al 2015), mais le taux de vaches atteintes reste non négligeable. La prévalence élevée avant 50 jours postpartum est attribuée au retard de la première ovulation (moins d'endométrites après la première ovulation chez la vache laitière qu'avant), la diminution importante ensuite pourrait être liée au bilan énergétique plus favorable à la guérison chez les vaches allaitantes que chez les vaches laitières (Santos et al 2009).

Si Santos et al (2009) n'observent pas d'effet délétères des endométrites sur la reproduction (observations sur 135 vaches Angus d'un seul élevage aux USA), pour Ricci et al (2015), l'intervalle vêlagesaillie fécondante est augmenté de 40 jours chez les vaches présentant une endométrite par rapport aux vaches saines (observations sur 97 vaches Piémontaises dans 6 élevages en Italie).

Les facteurs de variation de la prévalence des endométrites chez la vache allaitante n'ont pas été explorés à grande échelle à notre connaissance. Pour Ricci et al (2015), la prévalence des endométrites est plus élevée pour les vaches logées en stabulation entravée que pour les vaches logées en stabulation libre.

La qualité de l'involution utérine et la présence d'endométrite peuvent aussi être approchées par examen transrectal et échographique de l'utérus et des ovaires (d'après un score basé sur les structures ovariennes identifiées, le tonus de la paroi utérine, la taille de la lumière et la quantité de liquide présente dans l'utérus, Mee et al 2009). En utilisant cette technique, Carthy et al (2014) ont examiné plus de 40000 vaches laitières et allaitantes. L'involution utérine serait meilleure chez les vaches allaitantes que chez les vaches laitières $(78 \%$ de score 
1 (normal) en postpartum chez les premières contre $70 \%$ chez les secondes). La prévalence de scores élevés (involution anormale et/ou présence d'endométrite) augmente avec le rang de vêlage. Elle est plus élevée pour les vaches qui vêlent avec une aide difficile ou une césarienne que pour celles qui vêlent sans aide ou avec aide facile. La prévalence est plus élevée entre août et février qu'aux autres périodes de l'année, ce que les auteurs attribuent à une moins bonne couverture des besoins nutritionnels (observations réalisées en Irlande dans des systèmes herbagers) mais qui correspond aussi aux périodes où les vaches vêlent majoritairement en bâtiment plutôt qu'à l'herbe.
Il n'existe pas, à notre connaissance, de données françaises, ni sur les facteurs de risque, ni sur les conséquences des endométrites chez la vache allaitante. Ceci mériterait d'être exploré compte tenu de la fréquence élevée de cette affection.

\section{Conclusion}

La reproduction des vaches allaitantes françaises présente des particularités par rapport à celle des vaches laitières ou des vaches allaitantes de races étrangères plus précoces. Il existe de nombreux documents techniques actualisés annuellement qui font le point sur leurs performances mais certains aspects (prévalence des endométrites, fertilité notamment en monte naturelle) restent à explorer. Une bonne connaissance des particularités des races allaitantes françaises peut permettre d'améliorer les résultats technico-économiques des élevages et d'augmenter leur productivité. Une marge de progrès semble aussi exister pour l'utilisation et la valorisation des taureaux de monte naturelle, domaine qui a été peu exploré jusqu'à aujourd'hui.

\section{Remerciements}

Le groupe d'auteurs a été constitué pour réaliser cet article de synthèse à l'initiative du groupe MSD santé animale.

\section{Références}

Agabriel J., Grenet N., Petit M., 1992. État corporel et intervalle entre vêlages chez la vache allaitante. Bilan de deux années d'enquête en exploitation. INRA Prod. Anim., 5, 355-369.

Agabriel J., Blanc F., Egal D., D'Hour P., 2004. Influence combinées de la saison de mise bas et de l'exposition au taureau sur la venue en cyclicité de vaches Charolaises. Renc. Rech. Rum., 11, 398.

Aguer D., 1981. Les progestagènes dans la maîtrise des cycles sexuels chez les bovins. Rec. Med. Vet., 157, 53-60.

Beauchet S., 2010. Élaboration de recommandations pour la détection visuelle des chaleurs en troupeaux bovins allaitants. Thèse Médecine Vétérinaire, Oniris : École Nationale Vétérinaire Agroalimentaire et de l'Alimentation, Nantes, France, 121p.

Blanc F., Agabriel J., 2006. Intérêt de la modélisation pour interpréter l'influence de la date de vêlage sur la durée de l'anoestrus post-partum chez la vache adulte. Renc. Rech. Rum., 13, 277-280.

Blanc F., Dozias D., Agabriel J., 2002. Modélisation de l'efficacité de la reproduction chez la vache allaitante. Effet de la date d'introduction du taureau et de l'état d'engraissement au vêlage sur l'intervalle vêlage-saillie fécondante. Renc. Rech. Rum., 9, 65-68.

Blanc F., Bocquier F., Debus N., Agabriel J., D'Hour P., Chilliard Y., 2004. La pérennité et la durabilité des élevages de ruminants dépendent des capacités adaptatives des femelles. INRA Prod. Anim., 17, 287-302.

Blanc F., Paccard P., Gatien J., De La Torre A., Ponsart C., Egal D., Krauss D., Delval E., Agabriel J., 2010. Caractérisation de l'oestrus chez la vache allaitante: Quantification des manifestations comportementales et facteurs de variation. Renc. Rech. Rum., 17, 121-124.

Bo G.A., Baruselli P.S., 2014. Synchronization of ovulation and fixed-time artificial insemination in beef cattle. Animal, 8, 144-150.
Boivin X., Garel J.P., Le Neindre P., 1996. Importance du mode d'élevage dans le jeune âge ou lors du sevrage sur l'établissement des relations homme-animal chez les bovins allaitants et chez les caprins. Renc. Rech. Rum., 3 , 245-248.

Boivin X., Le Neindre P., Boissy A., Lensink J., Trillat G., Veissier I., 2003. Éleveur et grands herbivores : une relation à entretenir. INRA Prod. Anim., 16, 101-115.

Bovins Croissance, 2014. Résultats 2013 des élevages bovins viande suivis par Bovins Croissance. Institut de l'Élevage, Paris, France, $40 \mathrm{p}$.

Byerley D.J., Staigmiller R.B., Berardinelli J.G., Short R.E., 1987. Pregnancy rates of beef heifers bred either on puberal or third estrus. J. Anim. Sci., 65, 645-650.

Carthy T.R., Berry D.P., Fitzgerald A., McParland S., Williams E.J., Butler S.T., Cromie A.R. Ryan D., 2014. Risk factors associated with detailed reproductive phenotypes in dairy and beef cows. Animal, 8, 695-703.

Chanvallon A., Gatien J., Beauchet S., Salvetti P., 2014. La détection des chaleurs en troupeaux bovins allaitants. Renc. Rech. Rum., 21, 285.

Cobo E., Vinet A., Sapa J., Gallard Y., Desrame N., Dozias D., Phocas F., 2013. Relation entre puberté et croissance de la génisse charolaise. Renc. Rech. Rum., 20, 367.

Coutard J.P., 2011. Les génisses : l'avenir du troupeau allaitant. Chambre régionale d'agriculture des Pays de la Loire, Angers, 32p.

Coutard J.P., Menard M., Benoteau G., Lucas F., Henry J.M., Chaigneau F., Raimbault B., 2007. Reproduction des traupeaux allaitants dans les pays de la Loire : facteurs de variation des performances. Renc. Rech. Rum., 14, 359362 .

Crowe M.A., Diskin M.G., Williams E.J., 2014. Parturition to resumption of ovarian cyclicity: comparative aspects of beef and dairy cows. Animal, 8, 40-53.
Cutullic E., Delaby L., Causeur D., Michel G., Disenhaus C., 2009. Hierarchy of factors affecting behavioural signs used for oestrus detection of Holstein and Normande dairy cows in a seasonal calving system. Anim. Reprod. Sci., $113,22-37$

D'Hour P., Petit M., Garel J.P., 1996. Effet de la conduite alimentaire sur le développement et l'âge à la puberté de génisses Limousines et Salers. Renc. Rech. Rum., 3, 233-236.

Disenhaus C., Cutullic E., Blanc F., Gatien J., Agabriel J., Hetreau T., Michel G., Paccard P. Badinand F., Egal D., Ponsart C., 2008. Caractéristiques comparées de la cyclicité après vêlage de différentes races bovines. Renc. Rech. Rum., $15,383-386$

Dozias D., Agabriel J., Pecatte J.R., Petit M., 2006a. Influence du profil de croissance des génisses des troupeaux allaitants sur leurs performances ultérieures. Bull. GTV, Dijon, France 145-154.

Dozias D., Pecatte J.R., Michel G., 2006b. Influence du profil de croissance sur les performances de reproduction de génisses charolaises de 2 ans d'âge. Renc. Rech. Rum., 13, 287.

Ducrot C., Grohn Y.T., Humblot P., Bugnard F., Sulpice P., Gilbert R.O., 1994. Postpartum anestrus in French beef cattle: an epidemiological study. Theriogenology, 42, 753-764.

Esqurial H., Bouquier G., Goebels C., Roger O., Tesson C., Driancourt M.A., Fournier R., 2014. Comparaison de deux protocoles de synchronisation des chaleurs chez la vache allaitante : Les protocoles Crestar ${ }^{\circledR}$ et GPG+. Bull. GTV, Reims, France, 565-570.

Farrié J.P., Renon J., Bourge C., Gros J.M., Lahemade T., Muron G., Roudier J., 2008. Conditions et conséquences de la mise en place du vêlage à 2 ans dans un troupeau charolais. Renc. Rech. Rum., 15, 147-150.

Floch S., Deletang F., Freret S., Ponsart C., Remmy D., 2008. Control of oestrus with a Progesterone Intravaginal Device (Prid $\AA$ ): Comparison of 2 insertion durations. In: XX 
World Buiatrics Congress, Budapest, Hungary, 255 .

Floyd L.N., Lents C.A., White F.J., Wettemann R.P., 2009. Effect of number of cows in estrus and confinement area on estrous behavior of beef cows. J. Anim. Sci., 87, 1998-2004.

Fortin J., Coutard J.P., 2013. Choix et conséquences d'un premier vêlage à deux ans en élevages Charolais. Renc. Rech. Rum., 20, 246.

Gatien J., 2010. PRID $®$ Delta : Une IA 48 heures ou 56 heures après le retrait? Bulletin technique de l'Insémination Animale, 137, 33-35.

Grenet N., Dozias D., Billant J., 1996. Influence du niveau de croissance de génisses Charolaises et Limousines sur leur carrière de reproductrice. Renc. Rech. Rum., 3, 227-232.

Grimard B., Disenhaus C., 2005. Les anomalies de cyclicité après vêlage. Le Point Vétérinaire, $36,16-21$.

Grimard B., Humblot P., Mialot J.P., Ponter A.A., Sauvant D., Thibier M., 1994. Facteurs de variation de la durée de l'anoestrus postpartum et de la fertilité à l'oestrus induit chez la vache allaitante : importance du niveau d'apport énergétique. Renc. Rech. Rum., 1, 249252.

Grimard B., Humblot P., Ponter A.A., Chastant S., Constant F., Mialot J.P., 2003. Efficacité des traitements de synchronisation des chaleurs chez les bovins. INRA Prod. Anim., 16, 211-227.

Grimard B., Freret S., Gipoulou C., Delize F., Chambon G., Dewaele M., Driancourt M.A., Rosso V., Fournier R., Humblot P., Ponsart C., 2007. Fertilité à l'oestrus induit chez des génisses viande et des vaches allaitantes traitées à l'aide du nouveau protocole Crestar SO ${ }^{\circledR}$. Bull. GTV, 40, 71-78.

Guerrier J., Leudet O., 2014. Résultats du contrôle des perofrmances bovins allaitants. France, campagne 2013. Institut de l'Élevage, Paris, France, 107p.

Humblot P., Grimard B., 1996. Endocrinologie du post-partum et facteurs influençant le rétablissement de l'activité ovarienne chez la vache. Le Point Vétérinaire, 28, 73-81.

Humblot P., Grimard B., Ribon O., Khireddine B., Dervishi V., Thibier M., 1996. Sources of variation of post-partum cyclicity, ovulation and pregnancy rates in primiparous Charolais cows treated with norgestomet implants and PMSG. Theriogenology, 46, 1085-1096.

Hurnik J.F., King G.J., 1987. Estrous behavior in confined beef cows. J. Anim. Sci., 65, 431-438.

Institut de l'Élevage, 2014. Guide de l'alimentation du troupeau bovin allaitant. Institut de l'Élevage, Paris, France, 340p.

Institut de l'Élevage, Commission Nationale de l'Élevage, 2015. Chiffres clés des bovins 2014 Productions Lait et Viande. Institut de l'Élevage, Paris, France, 12p.

Landaeta-Hernandez A.J., Yelich J.V., Lemaster J.W., Fields M.J., Tran T., Chase C.C., Jr., Rae
D.O., Chenoweth P.J., 2002. Environmental, genetic and social factors affecting the expression of estrus in beef cows. Theriogenology, 57, 1357-1370.

Landaeta-Hernandez A.J., Melendez P., Bartolome J., Rae D.O., Archbald L.F., 2006. Effect of biostimulation on the expression of estrus in postpartum Angus cows. Theriogenology, 66, 710-716.

Lassalas J., Agabriel J., Petit M., 1996. Croissance de génisses Salers et performances en première lactation dans un système vêlage de printemps. Renc. Rech. Rum., 3, 255.

Le Cozler Y., Pecatte J.R., Porhiel J.Y., Brunschwig P., Disenhaus C., 2009. Pratiques d'élevage et performances des génisses laitières : état des connaissances et perspectives. INRA Prod. Anim., 22, 303-316.

LeBlanc S.J., 2014. Reproductive tract inflammatory disease in postpartum dairy cows. Animal, 8, 54-63.

Ledos H., Moureaux S., 2013. Durée de gestation pour les principales races de l'espèce bovine : Moyenne et variabilité. Institut de l'Élevage, Paris, France, 44p.

Mann G.E., Keatinge R., Hunter M., Hedley B.A., Lamming G.E., 2005. The use of milk progesterone to monitor reproductive function in beef suckler cows. Anim. Reprod. Sci., 88, 169-177.

Mee J.F., Buckley F., Ryan D., Dillon P., 2009. Pre-breeding ovaro-uterine ultrasonography and its relationship with first service pregnancy rate in seasonal-calving dairy herds. Reprod. Domest. Anim., 44, 331-337.

Meli C., 2009. Traitement de maîtrise des cycles à base de progestérone (CIDR $\left.{ }^{\circledR}\right)$ chez la vache : IA systématique ou sur chaleurs observées. Thèse Médecine Vétérinaire, Toulouse, France, 91p.

Mialon M.M., Renand G., Krauss D., Menissier F., 1995. Étude de la relation entre précocité sexuelle des génisses et croissance musculaire et poids testiculaire des taurillons en race Charolaise. Renc. Rech. Rum., 2, 201.

Mialon M.M., Renand G., Krauss D., Menissier F., 2000. Genetic variability of the length of postpartum anoestrus in Charolais cows and its relationship with age at puberty. Genet. Select. Evol., 32, 403-414.

Mialot J.P., Ponsart C., Gipoulou C., Bihoreau J.L., Roux M.E., Deletang F., 1998. The fertility of autumn calving suckler beef cows is increased by the addition of prostaglandin to progesterone and $\mathrm{eCG}$ estrus synchronization treatment. Theriogenology, 49, 1353-1363.

Mialot J.P., Constant F., Dezaux P., Grimard B Deletang F., Ponter A.A., 2003. Estrus synchronization in beef cows: comparison between GnRH+PGF2alpha+GnRH and PRID+PGF2 alpha+eCG. Theriogenology, 60, 319-330.

Miller S., 2014. Indicateurs Flux de reproducteurs Bovins Allaitants Campagne de naissance 2013. Institut de l'Élevage, Paris, France, 35p.
Montiel F., Ahuja C., 2005. Body condition and suckling as factors influencing the duration of postpartum anestrus in cattle: a review. Anim. Reprod. Sci., 85, 1-26.

Mounaix B., Ribaud D., David V., 2012. Vaccination contre la FCO : quel impact sur la fertilité des bovins allaitants? Renc. Rech. Rum., $19,138$.

Offredo T., Sibiluisky M., 2012. Facteurs déterminants de la productivité annuelle des élevages allaitants en Bretagne. Renc. Rech. Rum., 19, 358.

Petit M., Agabriel J., 1993. État corporel des vaches allaitantes Charolaises : signification, utilisation pratique et relations avec la reproduction. INRA Prod. Anim., 6, 311-318.

Phocas F., Sapa J., 2004. Genetic parameters for growth, reproductive performance, calving ease and suckling performance in beef cattle heifers. Anim. Sci., 79, 41-48.

Picard-Hagen N., Gayrard V., Saint-Blancat M., Ponsart C., 2011. Maîtrise des cycles chez la vache allaitante : Quels résultats en attendre ? Le Nouveau Praticien Vétérnaire, 4, 45-52.

Pierret P., Farrié J.-P., Renon J., 2009. Impact d'une conduite en vêlage à 2 ans en race Charolaise sur la morphologie et la réforme des vaches, la mortalité et la morbidité des veaux. Renc. Rech. Rum., 16, 383.

Recoules E., De La Torre A., Agabriel J., Egal D., Blanc F., 2013. Subcutaneous body lipids affect cyclicity and estrus behavior in primiparous Charolais cows. Anim. Reprod. Sci., 140, 115-123.

Ricci A., Gallo S., Molinaro F., Dondo A., Zoppi S., Vincenti L., 2015. Evaluation of subclinical endometritis and consequences on fertility in piedmontese beef cows. Reprod. Domest. Anim., 50, 142-148.

Roberson M.S., Wolfe M.W., Stumpf T.T., Werth L.A., Cupp A.S., Kojima N., Wolfe P.L., Kittok R.J., Kinder J.E., 1991. Influence of growth rate and exposure to bulls on age at puberty in beef heifers. J. Anim. Sci., 69, 20922098

Sanne E., Brouard S., Boulleau T., 2013. État des lieux et perspectives des filières viande bovine du Massif central Institut de l'Elevage, SIDAM Paris, France, 52p.

Santos N.R., Lamb G.C., Brown D.R., Gilbert R.O., 2009. Postpartum endometrial cytology in beef cows. Theriogenology, 71, 739-745.

Schillo K.K., Hall J.B., Hileman S.M., 1992. Effects of nutrition and season on the onset of puberty in the beef heifer. J. Anim. Sci., 70, 3994-4005.

Stagg K., Diskin M.G., Sreenan J.M., Roche J.F., 1995. Follicular development in long-term anoestrous suckler beef cows fed two levels of energy postpartum. Anim. Reprod. Sci., 38, 49-61.

Stagg K., Spicer L.J., Sreenan J.M., Roche J.F., Diskin M.G., 1998. Effect of calf isolation on follicular wave dynamics, gonadotropin and 
metabolic hormone changes, and interval to first ovulation in beef cows fed either of two energy levels postpartum. Biol. Reprod., 59, 777-783.

Troccon J.L., Petit M., 1989. Croissance des génisses de renouvellement et performances ultérieures. INRA Prod. Anim., 2, 55-64.

Veysset P., Benoit M., Laignel G., Bébin D., Roulenc M., Lherm M., 2014. Analyse et déterminants de l'évolution des performances d'élevages bovins et ovins allaitants en zones défavorisées de 1990 à 2012. In : Spécificités de l'élevage de ruminants en montagne. Grosclaude J., Thibier M., Baumont R. (Eds).Dossier, INRA Prod. Anim., 27, 49-64.

White F.J., Wettemann R.P., Looper M.L., Prado T.M., Morgan G.L., 2002. Seasonal effects on estrous behavior and time of ovulation in nonlactating beef cows. J. Anim. Sci., 80, 30533059 .
Yavas Y., Walton J.S., 2000a. Induction of ovulation in postpartum suckled beef cows: a review. Theriogenology, 54, 1-23.

Yavas Y., Walton J.S., 2000b. Postpartum acyclicity in suckled beef cows: a review. Theriogenology, 54, 25-55.

\title{
Résumé
}

La reproduction des vaches allaitantes présente des particularités par rapport à celle des vaches laitières. Améliorer leur reproduction est un levier pour augmenter leur productivité. Les génisses de race à viande sont pubères lorsqu'elles ont atteint 50-55\% du poids adulte vs $\mathbf{4 0 - 4 5} \%$ chez les génisses laitières. Les premières peuvent être mises à la reproduction vers $\mathbf{7 0} \%$ du poids adulte contre $60 \%$ pour les secondes. Le taux de renouvellement est assez faible en élevage allaitant, de 18 à $30 \%$ selon les races. Après le vêlage, la durée de l'anœstrus postpartum est plus longue qu'en race laitière du fait de la tétée du veau. L'expression des chaleurs est bonne chez la vache allaitante : les chaleurs durent entre 6 et 10 h dans les 3 principales races (Charolaise, Limousine et Blonde d'Aquitaine), et l'acceptation du chevauchement est exprimée dans 90 à $100 \%$ des œstrus. La monte naturelle domine en élevage allaitant : seuls 3 à $\mathbf{2 4} \%$ des veaux sont issus d'insémination selon les races. La fertilité des vaches allaitantes est actuellement mal connue, la performance de reproduction est plutôt approchée par la fécondité : l'intervalle vêlage-vêlage moyen des troupeaux est compris entre 379 et 444 jours selon les races. La proportion de vaches atteintes d'endométrite après vêlage semble élevée chez la vache allaitante comme chez la vache laitière (environ $\mathbf{2 0} \%$ même au-delà de $\mathbf{5 0}$ jours postpartum) mais les effets de cette affection sur la reproduction ont été peu explorés. De nombreuses données françaises concernant les races à viande spécialisées et rustiques sont présentées dans cet article.

\begin{abstract}
Specificities of reproduction in French breed suckler cows

Reproduction in beef cows is different compared to dairy cows. An increase in reproductive performance is a key factor by which to improve productivity in beef cows. Puberty occurs when beef heifers reach 50-55\% of their adult weight $v s .40-45 \%$ in dairy breeds. Beef heifers can be bred at $70 \%$ of their adult weight $v$ s. $60 \%$ for dairy heifers. Replacement rate is low in beef production, from 18 to $\mathbf{3 0} \%$ depending on the breed. Beef cows have a longer anostrous period after calving compared to dairy cows because the calf suckles its dam. The expression of oestrus is high in beef cows: the length of ostrus expression is between 6 and 10 hours in the 3 specialized French beef breeds (Charolaise, Limousine, Blonde d'Aquitaine) and 90 to $100 \%$ of cows express standing behavior at œstrous. Natural service is the most frequent breeding technique used in beef production: only 3 to $24 \%$ of calves are produced by artificial insemination depending on the breed. The fertility of beef cows is currently poorly understood. Reproductive efficiency is estimated by calving interval which is between 379 and $\mathbf{4 4 4}$ days depending on the breed. The prevalence of endometritis is high in both beef and dairy cows (levels of $20 \%$ have been recorded after 50 days postpartum) but its effect on reproductive performance has rarely been explored in beef cows. The present paper presents a large amount of French data concerning specialized and hardy French beef breeds.
\end{abstract}

GRIMARD B., AGABRIEL J., CHAMBON G., CHANVALLON A., CONSTANT F., CHASTANT S., 2017. Particularités de la reproduction des vaches allaitantes de races françaises. In : Élevage bovin allaitant. Agabriel J., Renand G., Baumont R. (Eds). Dossier, INRA Prod. Anim., 30, 125-138. 